\title{
Fabrication of fiber optic long period gratings operating at the phase matching turning point using an ultraviolet laser
}

\author{
Rebecca Y. N. Wong, Edmond Chehura, Stephen E. Staines, ${ }^{*}$ \\ Stephen W. James, and Ralph P. Tatam \\ Engineering Photonics, School of Engineering, Cranfield University, Cranfield MK43 OAL, UK \\ ${ }^{*}$ Corresponding author: s.w.james@ cranfield.ac.uk
}

Received 4 February 2014; revised 13 May 2014; accepted 16 May 2014; posted 12 June 2014 (Doc. ID 205985); published 14 July 2014

\begin{abstract}
It is known that optical fiber long period gratings (LPGs) exhibit their highest sensitivity to environmental perturbation when the period is such that the phase matching condition is satisfied at its turning point. The reproducible fabrication of LPGs with parameters satisfying this condition requires high resolution control over the properties of the grating. The performance of an LPG fabrication system based on the point-by-point UV exposure approach is analyzed in this paper, and the control of factors influencing reproducibility, including period, duty cycle, and the environment in which the device is fabricated, is explored. (C) 2014 Optical Society of America

OCIS codes: (060.2310) Fiber optics; (220.4610) Optical fabrication; (220.4830) Systems design; (060.2370) Fiber optics sensors; (060.2280) Fiber design and fabrication.

http://dx.doi.org/10.1364/AO.53.004669
\end{abstract}

\section{Introduction}

Optical fiber long period gratings (LPGs) are exploited in a range of applications, including as couplers to optimize the performance of pumping schemes in optical fiber lasers, as gain flattening filters, and as sensors [1-4]. LPGs offer a versatile platform for sensor development as a result of their sensitivity to strain, temperature, surrounding refractive index, and curvature [5]. In recent years there has been considerable interest in the development of chemical sensors with high sensitivity and specificity by coating the fiber with nanoscale coatings of functional materials [므료. For optimum sensitivity to environmental perturbation, it is known that the period of the LPG should be chosen such that the phase matching condition is satisfied at its turning point [9]. This places stringent requirements on the system used to fabricate the LPGs.

$1559-128 \mathrm{X} / 14 / 214669-06 \$ 15.00 / 0$

(C) 2014 Optical Society of America
An LPG is a periodic modulation of the propagation constants of the modes of an optical fiber, which promotes coupling of light between the core and cladding modes. LPGs typically have periods ranging from $100 \mu \mathrm{m}$ to $1 \mathrm{~mm}$. The coupling of light between the copropagating core and cladding modes occurs at discrete wavelengths and is dependent upon the product of the grating period with the difference between the effective refractive indices of the core mode and the cladding modes [10]:

$$
\lambda=\left[n_{\text {eff }}(\lambda)-n_{\text {clad }}^{i}(\lambda)\right] \Lambda,
$$

where $\lambda$ is the wavelength of the cladding mode, $n_{\mathrm{eff}}$ is the effective refractive index of the propagating core mode, $n_{\text {clad }}^{i}$ is the refractive index of the $i$ th cladding mode, and $\Lambda$ is the period of the LPG.

The modulation of the optical properties of the fiber may be achieved by modifying periodically the refractive index of the core by photoinduction or by physically deforming the optical fiber [1]. The 
refractive index may be modified by exposing locally the fiber to the output from a high-powered UV laser $[10,11]$, a $\mathrm{CO}_{2}$ laser $[12,13]$, an infrared femtosecond laser $[14,15]$, or by ion implantation [16]. Deformation of the fiber can be caused mechanically (e.g., inducing physical periodic corrugations on the fiber) [17] or by periodically tapering the fiber using a $\mathrm{CO}_{2}$ laser [18] or an electrical arc discharge [19] as the heat source.

The inscription of an LPG via UV irradiation can be carried out via a point-by-point method [20] or by using an amplitude mask [20]. An amplitude mask introduces a spatial modulation to the intensity of the laser beam incident on the optical fiber. In the pointby-point technique, the LPG is formed by exposing a section of length equal to half the period of the LPG to the UV beam for a set time and then translating either the fiber or the laser beam by a distance equal to the period, repeating the process until the desired length is attained. Although the fabrication of LPGs using the point-by-point approach can be more time consuming than using an amplitude mask, the flexibility of the technique allows the user to create LPGs with arbitrary period, with nonuniform period, and with arbitrary refractive index modulation profile by varying the dwell time at each point.

By calculating the dispersion of the core and cladding modes of the optical fiber, Eq. (1) can be used to generate a family of phase matching curves that predict the wavelengths at which energy will be coupled from the core to the cladding modes. Examples of the phase matching curves are shown in Fig. 1 , where the core and cladding mode refractive indices were determined using the approach presented originally in [21]. It can be seen that, for coupling to higher order modes, the phase matching curves contain a turning point.

It has been shown previously that, for coupling to a particular cladding mode, the sensitivity of the resonance band is a maximum when the period is chosen such that phase matching occurs at the phase matching turning point (PMTP). The evolution of the transmission spectrum of an LPG in response to, for example, changes in surrounding refractive index, under these conditions is characterized by the development of a broad resonance band that increases in extinction ratio with increasing difference between core and cladding mode effective refractive indices. As the surrounding refractive index increases further, the broad resonance band splits into two, with the dual resonance bands showing sensitivities of opposite sign. This is illustrated in Fig. 2 , where the evolution of the resonance band corresponding to coupling to the $\mathrm{LP}_{019}$ cladding mode in response to increasing the surrounding refractive index is simulated, using the approach described in [22] and [23], where the mode effective indices were calculated using the approach of [21], and the spectrum calculated using the matrix method of [24].

In addition to showing high sensitivity to environmental perturbation, the transmission spectra of

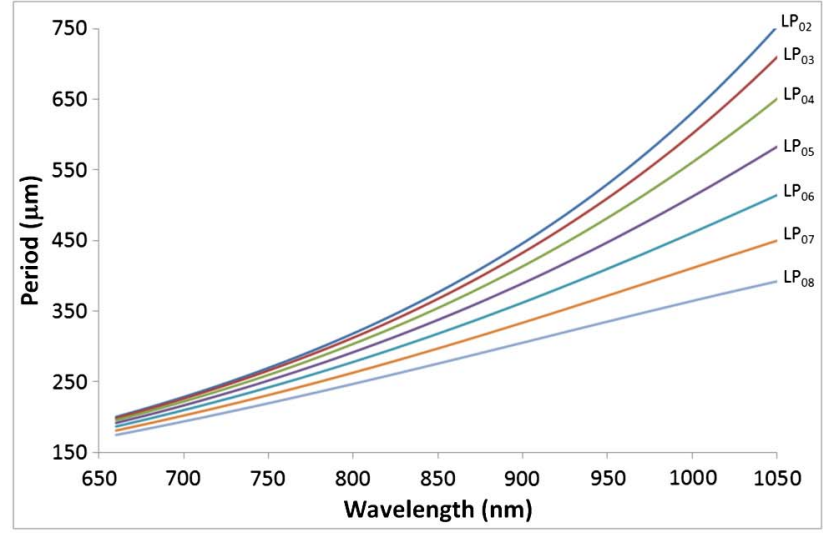

(a)

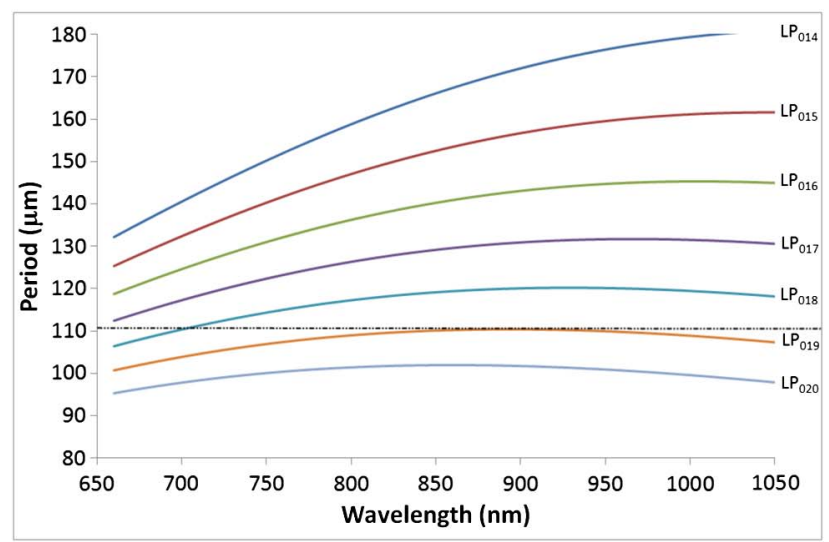

(b)

Fig. 1. (a) Phase matching curves for modes $\mathrm{LP}_{02}-\mathrm{LP}_{08}$. (b) Phase matching curves for modes $\mathrm{LP}_{014}-\mathrm{LP}_{020}$.

LPGs at the PMTP are also sensitive to the period, as can be seen from the evolution of the spectrum with increasing period shown in Fig. 3. Figure 3 shows that changes in period $<1 \mu \mathrm{m}$ have a significant influence on the spectrum. This sensitivity

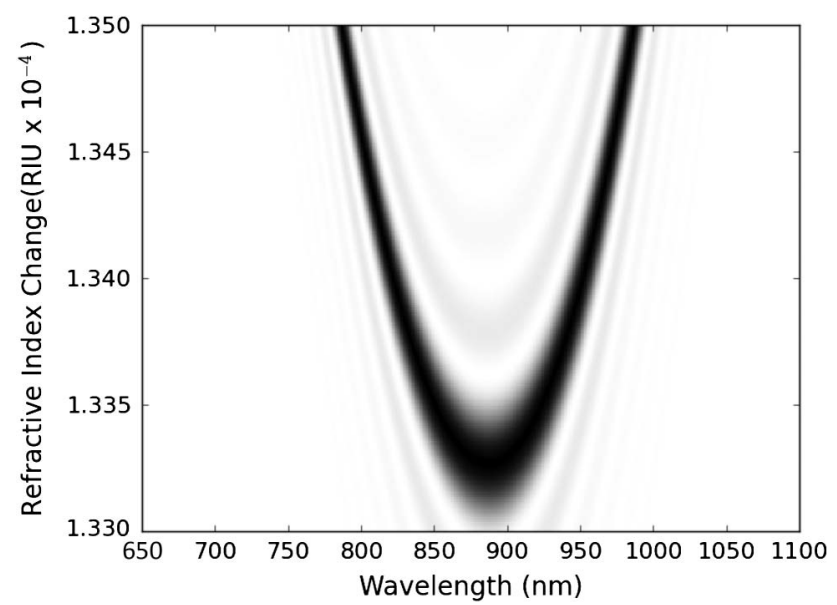

Fig. 2. Simulated evolution of the resonance band of an LPG of period $111 \mu \mathrm{m}$, corresponding to coupling to the $\mathrm{LP}_{019}$ cladding mode, in response to changes in surrounding refractive index. White and black represent 100\% and 0\% transmission, respectively. 


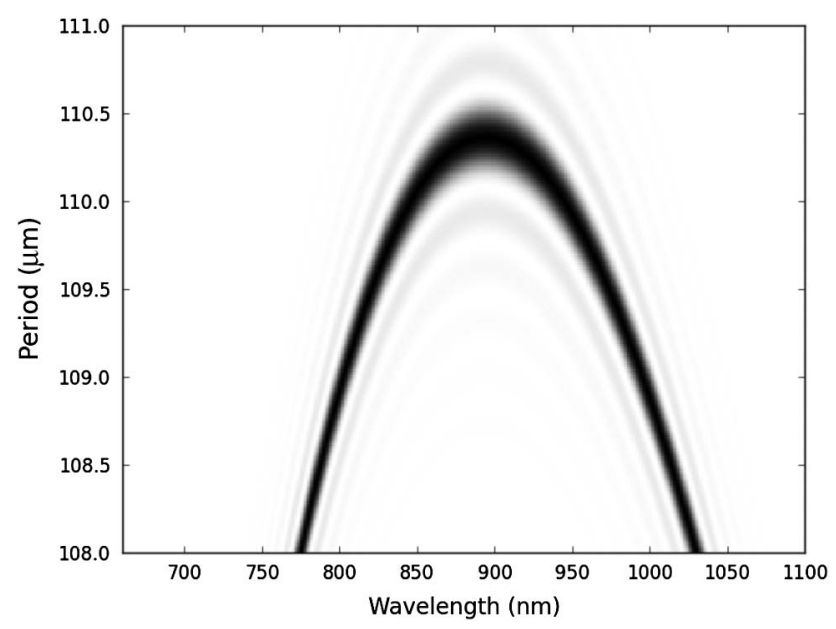

Fig. 3. Simulated evolution of the resonance band corresponding to coupling to the $\mathrm{LP}_{019}$ cladding mode in response to changes in grating period. White and black represent $100 \%$ and $0 \%$ transmission, respectively.

places stringent demands on the system used to fabricate LPGs.

Lan et al. [13] presented an investigation of the fabrication of LPGs at the PMTP using point-bypoint $\mathrm{CO}_{2}$ laser irradiation, exploring the influence of period and laser power on an LPG's transmission spectrum. It was suggested in [13] that the UV-laser fabrication of LPGs with period sufficiently short such that the coupling resonance occurs at the PMTP is usually undertaken using an amplitude mask, and that it requires postprocessing of the fiber to adjust the resonance wavelengths. Here we demonstrate that the point-by-point fabrication of LPGs using UV laser irradiation produces high-quality LPGs at the PMTP, and we investigate the influence of period, the duty cycle of the grating, the temperature of the environment in which the LPGs are fabricated, and the reproducibility of the fabrication process.

\section{LPG Fabrication}

\section{A. Optical Configuration}

The LPGs used in this study were fabricated using the point-by-point method. The output from a UV laser (injection-seeded, frequency-quadrupled, Spectra Physics Quanta-Ray Nd:YAG laser, repetition rate of $10 \mathrm{~Hz}$, output energy of $110 \mathrm{~mJ}$ at $266 \mathrm{~nm}$, pulse width 4-5 ns) was directed to the fiber by a series of optical components, as shown in Fig. 4. A variable attenuator was used to facilitate control over the laser power incident on the optical fiber.

The beam was directed toward a circular planoconvex lens of focal length $70 \mathrm{~mm}$, which was used to focus the beam down as it illuminated the fiber. Rather than using the lens to control the beam waist to define the length of the section of fiber illuminated, a slit was placed in front of the fiber, the width of which was adjusted by means of a computer controlled linear translation stage (PI, M-110.1DG), which has a resolution of $7 \mathrm{~nm}$ and maximum travel



Fig. 4. Optical setup used for fabricating LPGs using the pointby-point technique.

of $5 \mathrm{~mm}$. This allowed the length of the fiber exposed to be adjusted according to the desired period of the LPG. The slit width could be set to half the length of the desired period, ensuring a 50:50 duty cycle, or it could be adjusted such that any desired duty cycle could be achieved. The optical fiber was attached to a translation stage using v-groove fiber holders and magnets. The translation stage (PI, M-150.11) had a resolution of $8 \mathrm{~nm}$ and maximum travel of $50 \mathrm{~mm}$. The translation stages controlling the fiber movement and slit width were controlled in tandem by using a LabVIEW program that allowed the user to define the period, duty cycle, dwell time, chirp, LPG length, and, if desired, the location of any phase steps. The LPG's transmission spectrum was monitored by coupling light from a tungsten-halogen lamp into the proximal end of the fiber and connecting the distal end of the fiber to a CCD spectrometer (Ocean Optics HR4000) with a maximum optical resolution of $0.02 \mathrm{~nm}$.

\section{B. Fabrication Procedure}

The LPGs were inscribed in photosensitive fiber with a cutoff wavelength of $627 \mathrm{~nm}$ (Fibercore PS750), and all had a length of $35.000 \pm 0.003 \mathrm{~mm}$. Prior to exposure to the output from the laser, the polyacrylate jacket over the section of fiber to be exposed was removed by immersing it in a proprietary paint remover that contained dichloromethane. This softened the coating, which was then wiped off the fiber. The fiber was subsequently cleaned by wiping with a tissue soaked in isopropyl alcohol.

The temperature of the fabrication system was passively maintained at $24^{\circ} \mathrm{C}-24.5^{\circ} \mathrm{C}$ by placing the system in an insulated enclosure. A wireless temperature logger (i-button DS1923) was placed next to the fiber to monitor the temperature. The average power output of the laser was also monitored while fabricating each grating. This was carried out by monitoring the power of the light from the 
redundant output from a beam splitter placed in the beam path.

To investigate the influence of grating period on the transmission spectrum for an LPG designed to operate near the PMTP, a series of LPGs were fabricated with periods lying in the range of 110-111 $\mu \mathrm{m}$ and all with 50:50 duty cycle. The influence on the LPG transmission spectrum of changes in the grating period in the order of $100 \mathrm{~nm}$ was considered. Before each LPG was fabricated, the period was verified by placing a length of fiber with its polyacrylate buffer jacket intact into the system and exposing the fiber according to the programmed exposure profile. The exposed fibers, such as those shown in Fig. $\underline{5}$, were examined under an optical microscope and the period and duty cycle were measured.

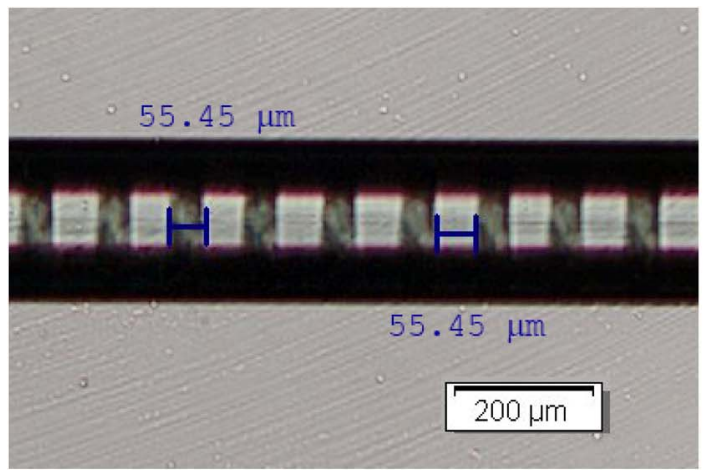

(a)

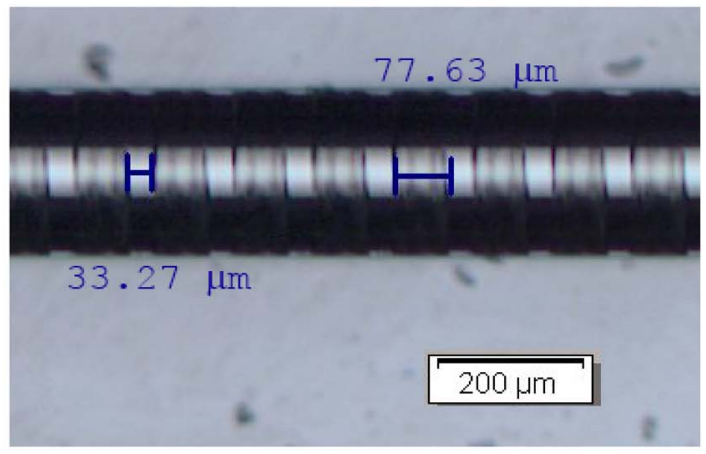

(b)

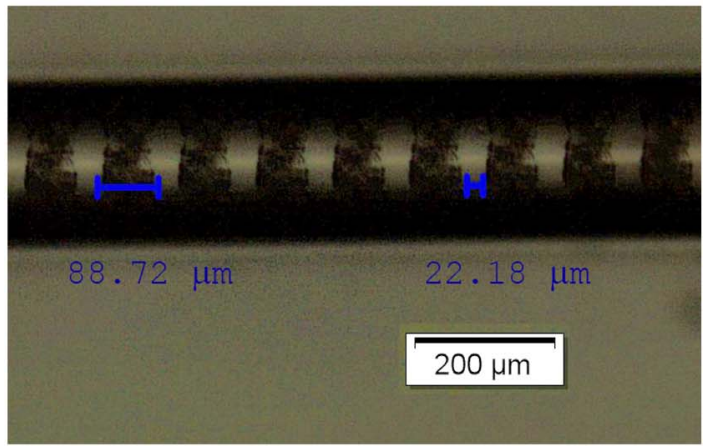

(c)

Fig. 5. Optical images of a $110.9 \mu \mathrm{m}$ period structure inscribed on the fiber buffer jacket of a single mode fiber with duty cycles (a) 50:50, (b) 70:30, and (c) 80:20. The darker sections denote the regions exposed to the laser through the slit.
Four LPGs, each of period $110.90 \pm 0.01 \mu \mathrm{m}$ and 50:50 duty cycle, were fabricated to investigate the reproducibility of the fabrication system setup. The period was selected to correspond with the PMTP for coupling to the $\mathrm{LP}_{019}$ mode. The investigation focused on the PMTP, as it is known that LPGs with periods in this region are highly sensitive to the surrounding environment, as discussed earlier. With the aim of investigating the influence of duty cycle on the LPG transmission spectrum, LPGs of period $110.70 \pm 0.01 \mu \mathrm{m}$ and $110.90 \pm 0.01 \mu \mathrm{m}$ were fabricated with varying duty cycles, namely 80:20, $70: 30$, and 50:50 (where the ratio is irradiated section: nonirradiated section). To explore the effect of changes in the temperature of the laboratory on the reproducibility of LPG fabrication, a number of LPGs of period $110.90 \pm 0.1 \mu \mathrm{m}$ were fabricated with the insulation removed.

\section{Results and Discussion}

Figure 6 illustrates the influence of the period on the spectra of LPGs around the PMTP. The $\mathrm{LP}_{019}$ mode resonance band shows a significant sensitivity to changes in the period of order $100 \mathrm{~nm}$. For example, there is a $45 \mathrm{~nm}$ change in the separation of the dual resonance bands when the grating period changes from 110 to $110.6 \mu \mathrm{m}$. The lower order modes, which at this period are further from their PMTPs, show successive reductions in sensitivity, as suggested by the analysis presented in Section 2 . This observation agrees with that reported in [13].

With the aim of studying the influence of duty cycle on the transmission spectrum, LPGs of period with differing duty cycles, $80: 20,70: 30$, and 50:50 (where the ratio is irradiated section: nonirradiated section), were fabricated. The results are shown in Figs. 7 and 8 for LPGs of period 110.7 and $110.9 \mu \mathrm{m}$, respectively. The resonance band extinction ratio was larger when the duty cycle was 50:50. It can be seen that varying the duty cycle of the fabrication process produces transmission spectra that make it appear that the LPG had a different period. This is due to a dependence of the local effective refractive index on the duty

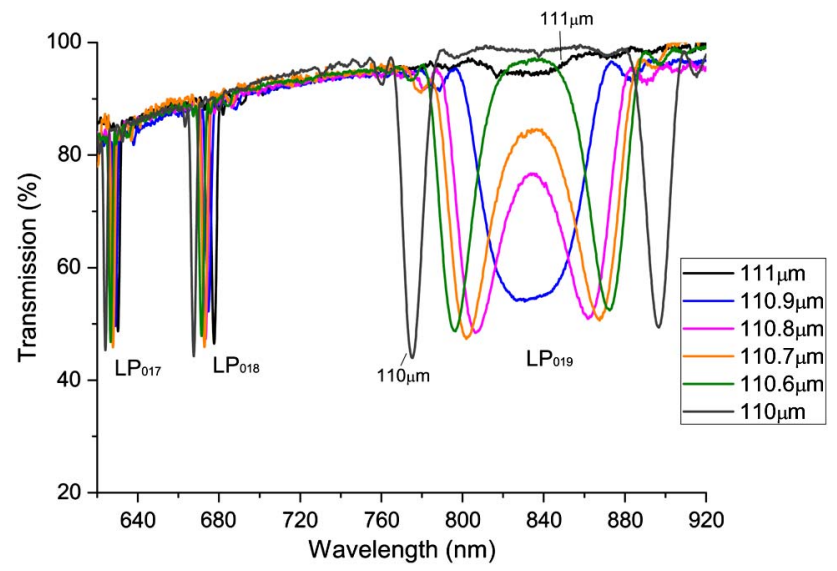

Fig. 6. Transmission spectra of LPGs written at and around the PMTP. 


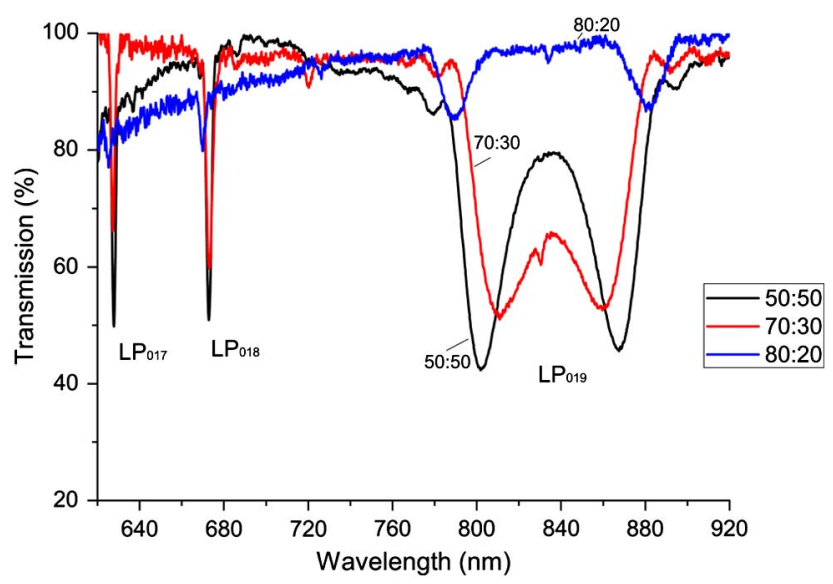

Fig. 7. LPG with a $110.7 \mu \mathrm{m}$ period with varying duty cycles of $50: 50,70: 30$, and $80: 20$ where the percentage ratio is irradiated section: nonirradiated section.

cycle. This was not explored by the authors of [13], where it would appear that the beam waist at the fiber was determined by the position of the focusing lens and was constant through their experiments.

The repeatability of the point-by-point LPG fabrication system was investigated by fabricating four LPGs of nominally identical period, $110.9 \mu \mathrm{m}$. The fabrication system was placed inside an insulated box to maintain the temperature within a $\pm 0.5^{\circ} \mathrm{C}$ range, corresponding to the resolution of the data logger used to monitor the temperature. The spectra are shown in Fig. 9 . The maximum difference between the resonance wavelengths recorded for the $\mathrm{LP}_{017}$ mode was $125 \pm 15 \mathrm{pm}$ and for the $\mathrm{LP}_{018}$ mode was also $125 \pm 15 \mathrm{pm}$. For the $\mathrm{LP}_{019}$ mode, the maximum change in transmission at the central wavelength of the band was from $42.4 \%$ to $36.3 \%$, a total change of $6.1 \% \pm 0.7 \%$. The slight change in the extinction of the resonance band could be due to a slight variation of environmental conditions or laser power. The laser power was observed to vary slowly by up to $3 \mathrm{~mW}$ over the duration of the fabrication process. Similar laser power differences

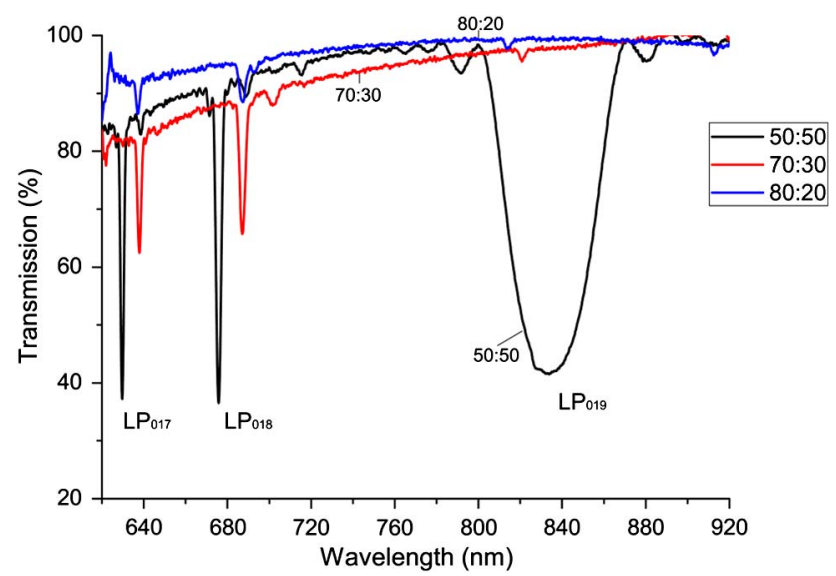

Fig. 8. LPG with a $110.9 \mu \mathrm{m}$ period with varying duty cycles of $50: 50,70: 30$, and $80: 20$ where the percentage ratio is irradiated section: nonirradiated section.



Fig. 9. Transmission spectra of four LPGs, each of $110.9 \mu \mathrm{m}$ period, fabricated in an environment in which the temperature was controlled to $0.5^{\circ} \mathrm{C}$.

investigated for $\mathrm{CO}_{2}$ laser fabrication [13] were observed to produce large difference in the transmission spectrum. This might be expected, as it was shown in [25] that the IR dose has a significant effect on the induced refractive index change, whereas this is a much smaller effect for UV irradiation [26].

To further illustrate the importance on the control of the environment in which the LPG is fabricated, the insulation was removed, such that the temperature varied by up to $5^{\circ} \mathrm{C} \pm 0.5^{\circ} \mathrm{C}$, and another five LPGs, each of period $110.9 \mu \mathrm{m}$, were fabricated. The extinction of the $\mathrm{LP}_{019}$ resonance band exhibited changes of up to $28.7 \% \pm 2.5 \%$ (from $74.9 \%$ to $46.2 \%$ ). The resonance bands corresponding to coupling to the $\mathrm{LP}_{017}$ and $\mathrm{LP}_{018}$ modes, away from the PMTP, exhibited maximum wavelength shifts of $390 \pm 43 \mathrm{pm}$ and $130 \pm 14 \mathrm{pm}$, respectively (Fig. 10).

Another issue that might influence the exact form of the spectrum is the tension that the fiber is placed under in its holder. From our experience of the fabrication of fiber Bragg gratings using similar configurations and methodologies to hold the fiber, we have found that the strain experienced by the optical



Fig. 10. Transmission spectra of four LPGs, each fabricated with a period $110.9 \mu \mathrm{m}$ where the temperature has not been controlled. 
fiber in the holder is of order $10 \mu \mathrm{m} / \mathrm{m}$, which would have a small influence on the transmission spectrum (changing the period by 1 part in $10^{5}$ ). It might be anticipated that this effect might be larger for $\mathrm{CO}_{2}$ laser-based inscription, as the tension might lead to fiber elongation (and thus a change in the period) as the fiber is heated by the $\mathrm{CO}_{2}$ laser [27].

\section{Conclusion}

We have demonstrated that the point-by-point fabrication of LPGs using UV laser irradiation produces high-quality LPGs at the PMTP. It has been shown that, in order to create reproducible gratings, a number of parameters have to be taken into account. These include the resolution of the translation stage used to move the fiber in front of the laser, control of the duty cycle, and the control of the temperature of the laboratory. The system is shown to have good reproducibility when the temperature is passively controlled; the depth of the $\mathrm{LP}_{019}$ mode has a variation of $6 \%$, but the central wavelength and mode profile remained unchanged. At the PMTP, changes of $<100 \mathrm{~nm}$ in the period of the LPG can lead to a large change in the transmission spectrum. The results confirm the observations reported on the fabrication of LPGs using a $\mathrm{CO}_{2}$ laser in [13] and expanded the fabrication parameters considered. The requirements on the fabrication system are relaxed considerably when fabricating LPGs with periods away from the PMTP.

We would like to thank the Engineering and Physical Sciences Research Council (EPSRC), UK for supporting this work via grant EP/H02252X. For inquiries relating to access to the research data or other materials referred to in this article, please contact Cranfield University Library and Information Services, library@cranfield.ac.uk.

\section{References}

1. F. Prudenzano, L. Messcia, T. Palmisano, M. Surico, M. De Sario, and G. C. Righini, "Optimization of pump absorption in MOF lasers via multi-long-period gratings: design strategies," Appl. Opt. 51, 1420-1430 (2012).

2. S. Baek, S. Roh, Y. Jeong, and B. Lee, "Experimental demonstration of enhancing pump absorption rate in claddingpumped ytterbium-doped fiber laser using pump coupling long-period gratings," IEEE Photon. Technol. Lett. 18, 700-702 (2006).

3. L. Mescia, "Design of long-period gratings in cladding-pumped microstructured optical fiber," J. Opt. Soc. Am. B 25, 1833-1839 (2008).

4. H. Sakata and K. Yamahata, "Variable long-period fiber gratings controlled by ND-FE-B permanent magnet for erbium-doped fiber sources," Microwave Opt. Technol. Lett. 56, 864-867 (2014).

5. S. W. James and R. P. Tatam, "Optical fiber long-period grating sensors: characteristics and application," Meas. Sci. Technol. 14, R49-R61 (2003).

6. T. Wang, S. Korposh, S. W. James, R. P. Tatam, and S.-W. Lee, "Optical fiber long period grating sensor with a polyelectrolyte alternate thin film for gas sensing of amine odors," Sens. Actuators. B 185, 117-124 (2013).

7. S. Korposh, T. Wang, S. W. James, R. P. Tatam, and S.-W. Lee, "Pronounced aromatic carboxylic acid detection using a layer-by-layer mesoporous coating on optical fiber long period grating, Sens. Actuators B 173, 300-309 (2012).

8. S. M. Topliss, S. W. James, F. Davis, S. P. J. Higson, and R. P. Tatam, "Optical fiber long period grating based selective vapor sensing of volatile organic compounds," Sens. Actuators B 143, 629-634 (2010).

9. C. S. Cheung, S. M. Topliss, S. W. James, and R. P. Tatam, "Response of fiber optic long period gratings operating near the phase matching turning point to the deposition of nanostructured coatings," J. Opt. Soc. Am. B 25, 897-902 (2008).

10. V. Bhatia and A. M. Vengsarkar, "Optical fiber long-period grating sensors," Opt. Lett. 21, 692-694 (1996).

11. J. Blows and D. Y. Tang, "Gratings written with tripled output of $Q$-switched Nd:YAG laser,” Electron. Lett. 36, 1837-1839 (2000).

12. D. D. Davis, T. K. Gaylord, E. N. Glytsis, S. G. Kosinski, S. C. Mettler, and A. M. Vengsarkar, "Long-period fiber grating fabrication with focused $\mathrm{CO}_{2}$ laser beams," Electron. Lett. 34, 302-303 (1998).

13. X. Lan, Q. Han, T. Wei, J. Huang, and H. Xiao, "Turn-aroundpoint long-period fiber gratings fabricated by $\mathrm{CO}_{2}$ laser pointby-point irradiations," IEEE Photon. Technol. Lett. 23, 1664-1666 (2011).

14. Y. Kondo, K. Nouchi, T. Mitsuyu, M. Watanabe, P. Kazansky, and K. Hirao, "Fabrication of long-period fiber gratings by focused irradiation of infra-red femtosecond laser pulses," Opt. Lett. 24, 646-648 (1999).

15. A. I. Kalachev, D. N. Nikogosyan, and G. Brambilla, "Longperiod fiber grating fabrication by high-intensity femtosecond pulses at $211 \mathrm{~nm}$," J. Lightwave Technol. 23, 2568-2578 (2005).

16. M. Fujumaki, Y. Ohki, J. L. Brebner, and S. Roorda, "Fabrication of long-period optical fiber gratings by use of ion implantation," Opt. Lett. 25, 88-90 (2000).

17. S. Savin, M. J. F. Digonnet, G. S. Kino, and H. J. Shaw, "Tunable mechanically induced long-period fiber gratings," Opt. Lett. 25, 710-712 (2000).

18. G. Kakarantzas, T. E. Dimmick, T. A. Birks, R. Le Roux, and P. S. J. Russell, "Miniature all-fiber devices based on $\mathrm{CO}_{2}$ laser microstructuring of tapered fibers," Opt. Lett. 26, 1137-1139 (2001).

19. G. Rego, O. Okhotnikov, E. Dianov, and V. Sulimov, "Hightemperature stability of long-period fiber gratings using an electric arc," J. Lightwave Technol. 29, 1137-1139 (2001).

20. L. Zhang, W. Zhang, and I. Bennion, "In-fiber grating optic sensors," in Fiber Optic Sensors, S. Yin, P. B. Ruffin, and F. T. S. Yu, eds., 2nd ed. (CRC Press, 2008), pp. 109-162.

21. E. Anemogiannis, E. N. Glytsis, and T. K. Gaylord, "Transmission characteristics of long-period fiber gratings having arbitrary azimuthal/radial refractive index variations," J. Lightwave Technol. 21, 218-227 (2003).

22. S. W. James, S. Korposh, S.-W. Lee, and R. P. Tatam, "A long period grating-based chemical sensor insensitive to the influence of interfering parameters," Opt. Express 22, 8012-8023 (2014).

23. S. W. James, S. M. Topliss, and R. P. Tatam, "Properties of length-apodized LPGs operating at the phase matching turning point," J. Lightwave Technol. 30, 2203-2209 (2012).

24. Y. Liu, J. A. R. Williams, L. Zhang, and I. Bennion, "Phase shifted and cascaded long-period fiber gratings," Opt. Commun. 164, 27-31 (1999).

25. Y. Li, T. Wei, J. A. Montoya, S. V. Saini, X. Lan, X. Tang, J. Dong, and $\mathrm{H}$. Xiao, "Measurement of $\mathrm{CO}_{2}$-laser-irradiationinduced refractive index modulation in single-mode fiber toward long-period fiber grating design and fabrication," Appl. Opt. 47, 5296-5304 (2008).

26. E. M. Dianov, S. A. Vasilev, O. I. Medvedkov, and A. A. Frolov, "Dynamics of the refractive index induced in germanosilicate optical fibres by different types of UV irradiation," Quantum Electron. 27, 785-788 (1997).

27. B. H. Kim, T.-J. Ahn, D. Y. Kim, B. H. Lee, Y. Chung, U.-C. Paek, and W.-T. Han, "Effect of $\mathrm{CO}_{2}$ laser irradiation on the refractive-index change in optical fibers," Appl. Opt. 41, 3809-3815 (2002). 


\title{
Fabrication of fiber optic long period
} gratings operating at the phase matching turning point using an ultraviolet laser

\author{
Wong, Rebecca Y. N
}

Optical Society of America

Wong RYN, Chehura E, Staines SE, et al., (2014) Fabrication of fiber optic long period gratings operating at the phase matching turning point using an ultraviolet laser. Applied Optics, Volume 53, Issue 21, July 2014, pp.4669-4674

https://doi.org/10.1364/AO.53.004669

Downloaded from Cranfield Library Services E-Repository 